\title{
ESTUDO DO COMPORTAMENTO DO CHUMBO EM LATOSSOLOS BRASILEIROS TRATADOS COM FOSFATOS: CONTRIBUIÇÕES PARA A REMEDIAÇÃO DE SÍTIOS CONTAMINADOS
}

\author{
Maria Luiza F. M. Kede* e Josino C. Moreira \\ Centro de Estudos da Saúde do Trabalhador e Ecologia Humana, Escola Nacional de Saúde Pública, Fundação Oswaldo Cruz, \\ Rua Leopoldo Bulhões, 1480, 21041-210 Rio de Janeiro - RJ, Brasil \\ Elena Mavropoulos e Alexandre M. Rossi \\ Centro Brasileiro de Pesquisas Físicas, Rua Dr. Xavier Sigaud, 150, 22290-180 Rio de Janeiro - RJ, Brasil \\ Luiz Carlos Bertolino \\ Universidade do Estado do Rio de Janeiro, Rua Francisco Portela, 794, 24435-000 Rio de Janeiro - RJ, Brasil \\ Daniel Vidal Perez \\ Embrapa Solos, Rua Jardim Botânico, 1024, 22460-000 Rio de Janeiro - RJ, Brasil \\ Nilce Carbonel Campos da Rocha \\ Instituto de Química, Universidade Federal do Rio de Janeiro, Cidade Universitária, CT, Bloco A, 21941-590 Rio de Janeiro - \\ RJ, Brasil \\ Recebido em 14/5/07; aceito em 17/8/07; publicado na web em 26/2/08

\begin{abstract}
STUDY OF LEAD BEHAVIOUR IN BRAZILLIAN LATOSOILS TREATED WITH PHOSPHATES: CONTRIBUTIONS TO THE REMEDIATION OF CONTAMINATED SITES. Phosphates have been used for lead immobilization in soils but the influence of soil type is not fully understood. In this work, lead chemical behaviour in two Brazilian latosoils (LA and LV) was studied via treatment with phosphates. The $\mathrm{Pb}$ concentration in Toxicity Characteristic Leaching Procedure (TCLP) solutions was decreased in all treatments. After treatment with $\mathrm{H}_{3} \mathrm{PO}_{4}$ the $\mathrm{Pb}$ concentration in the LA remained within the regulatory limit established by EPA. The ecotoxicological results with Daphnia pulex showed that this treatment reduced the lead bioavailability. Sequential extraction analyses showed that the lead was transferred from the most available to the residual fraction. Relevant decrease of soluble lead was observed in all phosphate treatments.
\end{abstract}

Keywords: remediation; lead; phosphates.

\section{INTRODUÇÃO}

Metais tóxicos são encontrados naturalmente no solo, em baixas concentrações, como resultado do intemperismo e de outros processos pedogenéticos. Entretanto, as concentrações naturais podem ser modificadas por processos biogeoquímicos e, principalmente, pela ação antrópica. Dentre esses metais, o chumbo merece destaque devido a suas características toxicológicas e a seu tempo de permanência nos solos. ${ }^{1}$ Este elemento pode permanecer nos solos sob forma relativamente estável devido à baixa solubilidade de suas formas químicas, por adsorção em componentes naturais, ${ }^{2}$ tais como a argila ou a matéria orgânica.

No Brasil existem várias áreas contaminadas com chumbo com reflexos sobre a saúde da população local. No Vale do Ribeira, localizado no norte do Paraná e sul de São Paulo, foi constatada a contaminação por chumbo em crianças residentes nas vizinhanças da empresa Plumblum que beneficiava e refinava minérios de chumbo. Enquanto o limite máximo de chumbo no sangue de crianças recomendado pela Organização Mundial da Saúde (OMS) é de $10 \mu \mathrm{g} \mathrm{dL}^{-1}$, nesta região já foram registradas concentrações de até $37,8 \mu \mathrm{g} \mathrm{dL}^{-1}$. 3

No município de Santo Amaro da Purificação-BA, onde se encontram antigas instalações de outra filial da empresa Plumblum, foi constatada alta contaminação por chumbo em boa parte da população, no solo e nos sedimentos do rio Subaé. O uso de escória para aterros e para a construção de casas contribuiu para elevar a extensão da contaminação. ${ }^{4}$

\footnotetext{
*e-mail: luiza.kede@ensp.fiocruz.br
}

Em Bauru-SP, a indústria de baterias automotivas Ajax foi responsável por outro exemplo de contaminação humana e ambiental. Análises realizadas nos laboratórios da Companhia de Tecnologia de Saneamento Ambiental (CETESB) ${ }^{5}$ detectaram concentrações extremamente elevadas de chumbo na atmosfera, registrando valores de até $37,7 \mu \mathrm{g} / \mathrm{m}^{3}$, que superam o padrão adotado por esta agência ambiental que corresponde a $1,5 \mu \mathrm{g} / \mathrm{m}^{3}$.

Devido as suas propriedades bioquímico-fisiológicas sobre os organismos vivos, o chumbo quando incorporado ao ambiente ou aos organismos constitui ameaça à saúde do homem e dos ecossistemas. A maior parte dos casos de envenenamento por chumbo ocorre por ingestão oral e a absorção acontece no intestino. Uma vez absorvido, o chumbo é distribuído através do sangue para os diversos órgãos e sistemas e cerca de $90 \%$ se deposita nos ossos, onde substitui o cálcio. Esta absorção é maior em crianças que em adultos. $^{6}$

As crianças são expostas ao chumbo por uma variedade de fontes ambientais, mas, fundamentalmente pelo hábito de levar a mão à boca. Os fetos e as crianças menores de 7 anos constituem os grupos de maior risco às ações maléficas do chumbo. Esse metal atravessa facilmente a placenta produzindo neurotoxicidade, afetando diferentes atividades biológicas em níveis celular e molecular podendo comprometer a função cognitiva e o comportamento das crianças. ${ }^{7}$ Entretanto, a toxicidade dos metais depende de sua forma química, ou seja, de sua especiação química, que está intimamente relacionada à sua biodisponibilidade. Assim, diferentes espécies químicas do chumbo podem apresentar toxicidades diferentes e, normalmente, as formas mais solúveis são mais biodisponíveis. ${ }^{8}$ 
Medidas utilizadas para diminuir a solubilidade e a biodisponibilidade do chumbo presente em amostras ambientais têm sido propostas como forma de diminuir os impactos desta contaminação sobre populações expostas. Alguns autores ${ }^{9}$ têm mostrado que a remediação de solos contaminados pela adição de fosfatos é eficiente na transformação de chumbo do suas formas químicas mais solúveis em formas menos solúveis (residuais). A eficácia desta imobilização é atribuída à formação de espécies minerais insolúveis, semelhantes à piromorfita que se observa após a aplicação dos fosfatos. Entretanto, estes estudos foram desenvolvidos utilizando solos do domínio temperado que possuem propriedades químicas e físicas diferenciadas daquelas do clima tropical.

O Brasil está situado numa região climática tropical úmida, tendo como solos mais importantes os latossolos que ocorrem em quase todas as regiões do país. Os latossolos são geralmente solos mais velhos e profundos, bastante intemperizados, lixiviados e com baixa fertilidade.

Com o objetivo de entender mais detalhadamente o comportamento do chumbo nestes latossolos e conseqüentemente poder contribuir para a remediação dos sítios contaminados no Brasil, dois tipos de latossolos brasileiros, um Amarelo (LA) e outro Vermelho (LV), foram contaminados com chumbo e tratados com fosfatos.

\section{PARTE EXPERIMENTAL}

\section{Materiais e métodos}

Amostras de latossolos foram coletadas, em uma profundidade média de $10 \mathrm{~cm}$, em Lavras (MG) e no Rio de Janeiro. Estas amostras foram secas em estufa, destorroadas e peneiradas ( $2 \mathrm{~mm})$. Suas propriedades químicas e físicas (granulometria, mineralogia de argila, teor de matéria orgânica, capacidade de retenção de água, capacidade de troca cationnica - $\mathrm{CTC}$ e $\mathrm{pH}$ ) foram determinadas segundo metodologia recomendada pela EMBRAPA. ${ }^{10}$

Os solos foram contaminados com $\mathrm{Pb}\left(\mathrm{NO}_{3}\right)_{2}$, homogeneizados, colocados em vasos de $2 \mathrm{~kg}$ e acondicionados em ambiente controlado na casa de vegetação da Embrapa Solos - RJ. A concentração final determinada foi em média de $7000 \mathrm{mg} \mathrm{kg}^{-1}$. Todas as amostras de solo foram mantidas úmidas a $80 \%$ da capacidade de campo.

Depois de um período de incubação de 5 meses, os solos foram submetidos a 3 tipos de tratamentos com fosfatos: $\mathrm{T} 1 \mathrm{H}_{3} \mathrm{PO}_{4}, \mathrm{~T} 2$ rocha fosfatada (RF) e T3 uma mistura de $\mathrm{H}_{3} \mathrm{PO}_{4}$ e $\mathrm{RF}$ numa razão molar $\mathrm{P} / \mathrm{Pb}$ de $6: 1$ para os três tratamentos. Os controles de ambos LA e LV foram denominados de T0. Cada tratamento, incluindo o controle, foi realizado em 5 repetições totalizando 20 vasos para cada tipo de solo.

$\mathrm{O}_{3} \mathrm{PO}_{4}$ utilizado $(85 \% \mathrm{v} / \mathrm{v})$ foi obtido da Vetec. A rocha fosfatada em forma de pó heterogêneo foi homogeneizada em gral e classificada granulometricamente em seis frações $(>0,250 ; 0,210$ $0,250 ; 0,177-0,210 ; 0,125-0,177 ; 0,074-0,125 ;<0,074 \mathrm{~mm})$ com o objetivo de determinar a composição mineralógica nos diferentes intervalos granulométricos.

Os picos dos difratogramas de raios-X (Figura 1) mostram que os minerais identificados como apatitas (Ap) fazem parte de um grupo e são representados por uma série isomórfica constituída por fluorapatita $\left(\mathrm{Ca}\left(\mathrm{PO}_{4}\right)_{3} \mathrm{~F}\right)$, carboapatita $\left(\mathrm{Ca}_{5}\left(\mathrm{PO}_{4}, \mathrm{CO}, \mathrm{OH}\right)_{3}(\mathrm{FOH})\right.$. Nos minerais deste grupo o flúor, o carbonato e a hidroxila podem substituir-se mutuamente. ${ }^{11}$

Através dos difratogramas de raios- $\mathrm{X}$ observou-se que a composição mineralógica das várias frações granulométricas se altera. As frações mais finas são enriquecidas em apatita. Experimentos de sorção constataram que $0 \mathrm{~Pb}^{+2}$ em solução é principalmente imobilizado por frações mais finas da rocha. ${ }^{12}$ Dessa forma, optou-

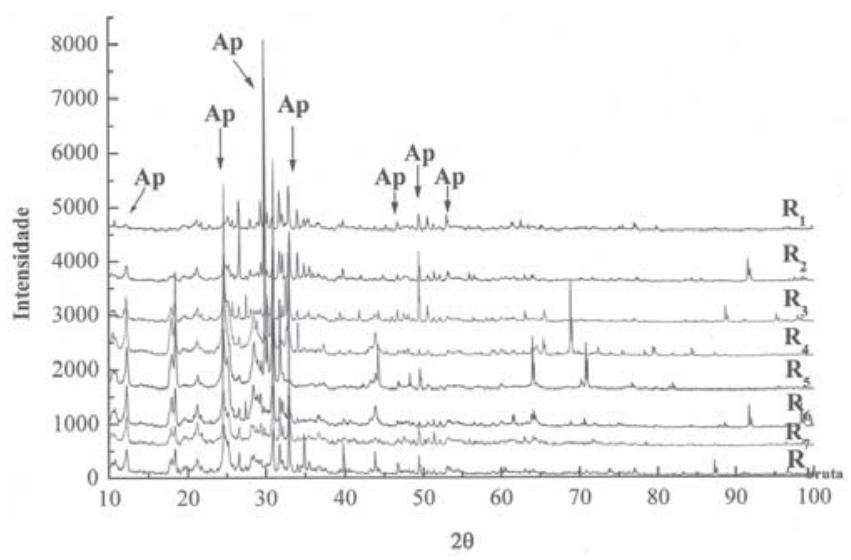

Figura 1. Difratogramas de raios- $X$ das amostras peneiradas $(R 1<0,074$; $R 2$ 0,074-0,125; R3 0,125-0,177; R4 0,177-0,210; R5 0,210-0,250; R6 $>0,250 ; R 7<0,210$ e $R 8$ rocha bruta)

se por trabalhar com as frações mais finas, que possuem uma quantidade maior de apatitas.

Foram coletadas subamostras após 60, 120 e 270 dias para a avaliação do potencial toxicológico dos solos através do teste "Toxicity Characteristic Leaching Procedure (TCLP)", 13 a mobilidade do metal foi analisada por extração seqüencial e as variações de toxicidade nos solos LA e LV foram avaliadas através do teste de ecotoxicidade com Daphnia pulex. ${ }^{14}$

\section{TCLP ("Toxicity Characteristic Leaching Procedure")}

O TCLP é um método recomendado pela EPA para definir o grau de perigo que um material em particular oferece à saúde. $\mathrm{O}$ limite máximo de chumbo na solução extratora (TCLP) é de $5 \mathrm{mg}$ $\mathrm{Pb} / \mathrm{L}$ e acima desse valor os rejeitos são considerados perigosos.

O fluido extrator ( $\mathrm{pH}$ entre 4,93 $\pm 0,05$ ) foi preparado com 5,7 $\mathrm{mL}$ de ácido acético para $500 \mathrm{~mL}$ de água, mais $64,3 \mathrm{~mL} \mathrm{NaOH}$ $1 \mathrm{~N}$ e diluído em um volume de $1 \mathrm{~L}$.

Nesse estudo a metodologia utilizada pela EPA foi modificada. Para a preparação da amostra 2,5 g de cada tipo de solo foram pesados em triplicata e reduzidos a partículas de $1 \mathrm{~mm}$. As amostras foram acondicionadas em tubos de $50 \mathrm{~mL}$ e acidificadas com fluido extrator. Os tubos foram colocados em um agitador Kliner NT 150 a $10 \mathrm{rpm}$ durante $18 \mathrm{~h}$ em temperatura ambiente. Após esse período de agitação as amostras foram centrifugadas (Excelsa Baby II Modelo 206- R) e filtradas e as concentrações de chumbo determinadas por ICP-OES modelo OPTIMA 3000 - Perkin-Elmer.

\section{Extração seqüencial}

O método de extração seqüencial ${ }^{15}$ foi utilizado para avaliar o comportamento do chumbo nos solos. Neste estudo optou-se pelo protocolo recomendado por Tessier. ${ }^{16}$ De acordo com o procedimento apresentado na Figura 2, as formas químicas dos metais presentes na amostra são classificadas em fração solúvel em água (F1), fração trocável (F2), fração ligada a carbonato (F3), fração ligada a óxidos de ferro e manganês $(\mathrm{F} 4)$, fração ligada à matéria orgânica (F5) e fração residual (F6). As frações 1 -3 são mais facilmente solúveis e assim podem ser incorporadas mais facilmente pela biota, enquanto as frações 4 a 6 são menos solúveis e, portanto, ambientalmente mais estáveis.

Este procedimento foi realizado retirando-se subamostras do solo, em triplicata, de cada um dos 5 vasos contendo solos tratados e não tratados com fosfatos. Após cada extração as amostras foram centrifugadas a $6000 \mathrm{rpm}$ por $30 \mathrm{~min}$. 


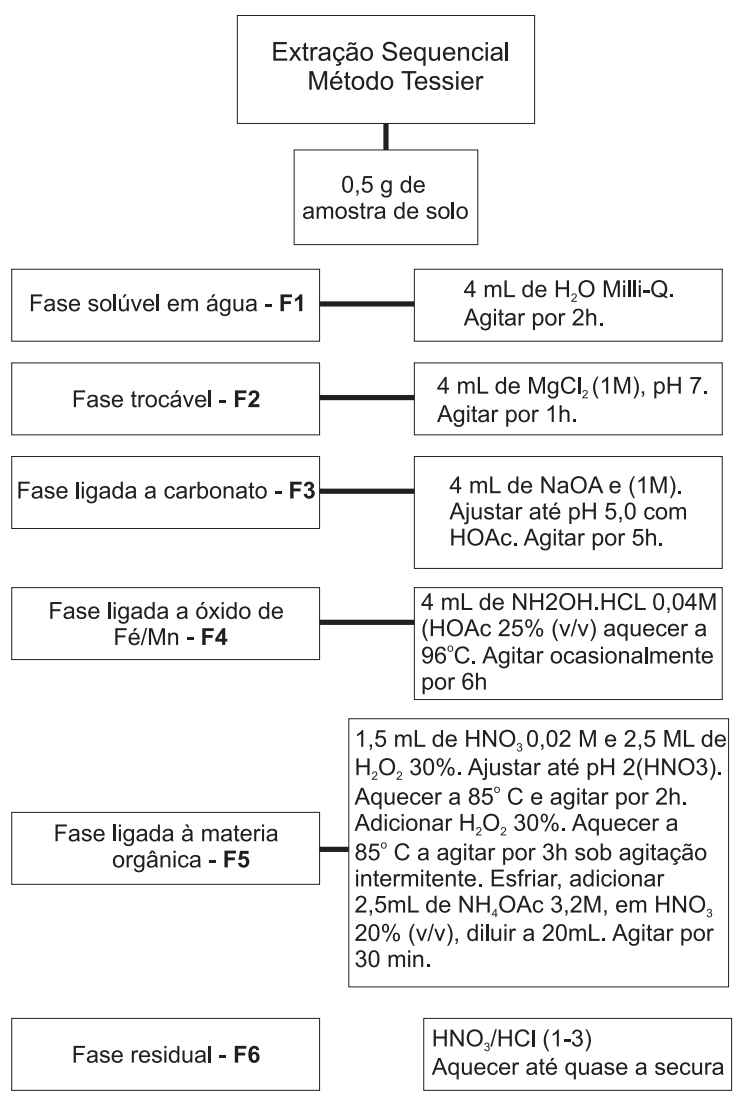

Figura 2. Fluxograma com as etapas e os reagentes utilizados na extração seqüencial segundo Tessier

\section{Métodos estatísticos}

Para as análises estatísticas, dos resultados de TCLP e extração seqüencial, foram usadas a análise de variância (ANOVA) utilizando o programa estatístico SAEG $^{17}$ e o teste de comparação de médias Tukey ou Scott Knott, o qual faz agrupamento estatístico a partir das médias. ${ }^{18}$

Por se tratar de um experimento em vasos o delineamento foi inteiramente casualizado, com cinco repetições e cada extração e determinação de chumbo foi realizada em triplicata.

\section{Teste ecotoxicológico com Daphnias pulex}

O teste de ecotoxicidade com Daphnia pulex ${ }^{14}$ foi realizado após 460 dias de tratamento com fosfatos. Este teste consiste em expor Daphnias jovens (2 a $26 \mathrm{~h}$ de vida) a várias diluições de uma amostra, por um período de $24 \mathrm{~h}$ e observar o comportamento destes organismos após este tempo. A metodologia para preparo da solução e execução do teste foi recomendada por Hyötyläinen e Oikari. ${ }^{19}$
Para a preparação das soluções-teste foram pesados $20 \mathrm{~g}$ de solo, usando alíquotas de 2 vasos para cada tratamento, totalizando 10 amostras para cada tipo de solo. Depois foram adicionados 80 $\mathrm{mL}$ de água (água mineral Minalba) em cada bequer, perfazendo uma razão de 1:4. Estas misturas foram agitadas por $1 \mathrm{~h}$ e mantidas em geladeira a $4{ }^{\circ} \mathrm{C}$ por $24 \mathrm{~h}$, para que o material em suspensão pudesse decantar. Após esse tempo as soluções foram filtradas com filtro de papel e congeladas até o momento do teste.

\section{RESULTADOS E DISCUSSÃO}

As características físicas e químicas dos solos estudados são mostradas na Tabela 1. Os solos LA e LV possuem textura argilosa e muito argilosa, respectivamente, segundo a $\mathrm{EMBRAPA}^{10}$ e são bastante distintos quando se comparam os valores de CTC e matéria orgânica. O LV possui valor de CTC 5 vezes maior que o LA e matéria orgânica aproximadamente 13 vezes mais elevada. Essas diferenças nas características dos solos são de fundamental importância para o entendimento dos mecanismos envolvidos no processo de remediação do chumbo através de fosfatos.

Os pHs dos solos antes da adição do chumbo já eram ácidos, permanecendo em torno de 5,0 para o solo LA e 4,5 para o LV. Após a contaminação os pHs dos solos LA e LV decrescem para 4,16 e 3,36 , respectivamente. Isto é explicado pelo processo de hidrólise do chumbo em água formando $\mathrm{Pb}(\mathrm{OH})_{2}$ e liberando $\mathrm{H}^{+}{ }^{20}$ Como esperado, os pHs dos dois solos variaram após os tratamentos com fosfatos (Figura 3).

A rocha fosfatada utilizada neste trabalho apresentava composição mineralógica variável, mas as frações mais finas eram enriquecidas em apatita. ${ }^{12}$ Dessa forma, optou-se por utilizar as frações mais finas, cuja composição química é mostrada na Tabela 2.

\section{Teste de TCLP ("Toxicity Characteristic Leaching Procedure")}

Os dados obtidos nos testes TCLP foram submetidos à análise de variância (ANOVA) utilizando o programa estatístico SAEG ${ }^{17}$ com base nos valores absolutos (mg de $\mathrm{Pb} \mathrm{kg}^{-1}$ de solo). Em todos os solos tratados com fósforo ocorreu decréscimo dos níveis de chumbo na solução de TCLP quando comparados ao controle (Figuras $4 \mathrm{a}$ e $4 b)$.

A diminuição mais significativa, para os dois solos, aconteceu no $\mathrm{T} 1\left(\mathrm{H}_{3} \mathrm{PO}_{4}\right)$, seguido pelo $\mathrm{T} 3\left(\mathrm{H}_{3} \mathrm{PO}_{4}+\mathrm{RF}\right)$ e por último no $\mathrm{T} 2$ (RF). Os resultados do T1 para o LA ficaram abaixo do nível regulatório com decréscimo médio de 99,2\% quando comparado ao T0, para coleta 1 (60 dias), 2 (120 dias) e 3 (270 dias) após os tratamentos. O T3 no solo LA, apesar de não ter ficado abaixo do limite de chumbo para o TCLP estipulado pela EPA, também obteve um grande decréscimo da concentração desse metal, em torno de $94,9 \%$. O T2 foi o tratamento que menos reduziu o valor de chumbo, em média $26,6 \%$ para as três coletas.

Comparando-se ao T0 e considerando as três coletas, o solo LV no T1 conseguiu reduzir em média $80,1 \%$ a concentração de chumbo, seguido pelo T3 com 57,8\% e T2 com 10,2\%.

Tabela 1. Características físicas e químicas dos solos LA e LV

\begin{tabular}{|c|c|c|c|c|c|c|c|}
\hline \multirow[t]{2}{*}{ Solos } & \multicolumn{3}{|c|}{$\begin{array}{c}\text { Composição granulométrica } \\
\text { da terra fina }(\mathrm{g} / \mathrm{kg})\end{array}$} & \multirow[b]{2}{*}{$\begin{array}{l}\mathrm{Fe}_{2} \mathrm{O}_{3} \\
\left(\mathrm{~g} \mathrm{~kg}^{-1}\right)\end{array}$} & \multirow[b]{2}{*}{$\begin{array}{c}\mathrm{MnO} \\
\left(\mathrm{mg} \mathrm{kg}^{-1}\right)\end{array}$} & \multirow[b]{2}{*}{$\begin{array}{c}\text { CTC } \\
\left(\mathrm{cmolc} \mathrm{kg}^{-1}\right)\end{array}$} & \multirow[b]{2}{*}{$\begin{array}{c}\text { Carbono } \\
\text { orgânico }\left(\mathrm{g} \mathrm{kg}^{-1}\right)\end{array}$} \\
\hline & Areia & Silte & Argila & & & & \\
\hline LA & 366 & 154 & 480 & 47,4 & 72,5 & 3,1 & 2,6 \\
\hline LV & 198 & 162 & 640 & 104,0 & 273,0 & 15,5 & 34,4 \\
\hline
\end{tabular}



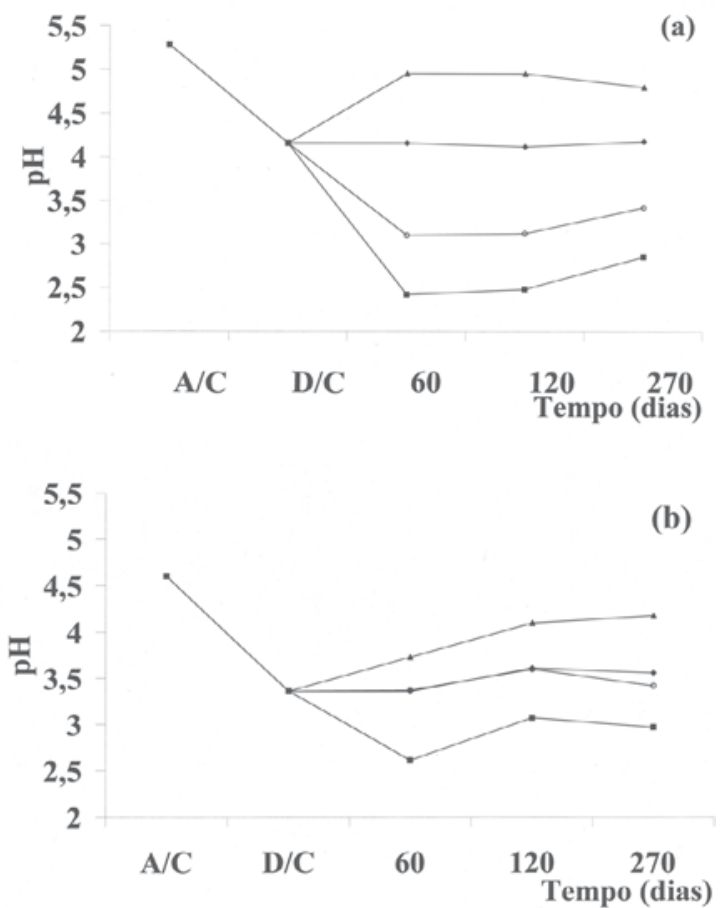

Figura 3. Valores do $p H$ antes da contaminação $(A / C)$, depois da contaminação com chumbo $(D / C), 60,120$ e 270 dias após os tratamentos: TO (•); T1 (ם);T2 ( $\mathbf{\Delta})$ e T3 (O). LA (a) e LV (b)

Tabela 2. Composição química da rocha fosfatada

\begin{tabular}{lccccccc}
\hline Amostra & & \multicolumn{3}{c}{ Análise por } & \multicolumn{3}{c}{ Análise } \\
& & \multicolumn{3}{c}{ via úmida $(\%)$} & & \multicolumn{2}{c}{ FRX (\%) } \\
& $\mathrm{P}_{2} \mathrm{O}_{5}$ & $\mathrm{Fe}_{2} \mathrm{O}_{3}$ & $\mathrm{CaO}$ & $\mathrm{MgO}$ & $\mathrm{Al}_{2} \mathrm{O}_{3}$ & $\mathrm{TiO}_{2}$ & $\mathrm{SiO}_{2}$ \\
\hline $\begin{array}{l}\text { Rocha } \\
\text { fosfatada } \\
<0,02 \mathrm{~mm}\end{array}$ & 11,08 & 19,22 & 16,27 & 4,22 & 3,15 & 7,06 & 21,98 \\
\hline
\end{tabular}

Através da comparação entre os tratamentos em cada solo teremos o maior valor de concentração de chumbo liberado, no LA, para T0, depois T2. O T1 e o T3 não possuem diferenciação estatística, ou seja, não diferem entre si nas três coletas. Para o LV nas coletas 1 e 2 observamos uma concentração maior de chumbo no T0, depois T2. T1 e T3 não se diferenciam significativamente. Na coleta 3 não há diferenciação significativa entre todos os tratamentos para o LV.

As diferenças entre os valores de chumbo obtidos após os tratamentos podem estar associadas à velocidade com que as reações ocorrem nos solos, devido às diferentes fontes de fosfato. Sabe-se que a eficiência do tratamento utilizando fosfatos depende da habilidade da fonte de fosfato em fornecer fósforo solúvel ao meio. ${ }^{21}$ Quanto maior a velocidade da disponibilização do fósforo para o meio, mais rapidamente a fase estável será formada. Comparando-se os três tratamentos estudados, são esperadas reações mais rápidas quando o $\mathrm{H}_{3} \mathrm{PO}_{4}$ é utilizado. Isto acontece porque o ácido é uma fonte solúvel de fósforo, enquanto que a rocha sendo uma fonte sólida necessita de um tempo maior para disponibilizar o fósforo.

O LV mostrou-se mais ativo que o LA na imobilização do chumbo sugerindo que as maiores concentrações de óxidos de Fe e Mn e de matéria orgânica competem com o fósforo pelo chumbo, o que não ocorre com LA, que é um solo pouco reativo por ser essencialmente caulinítico.

Comparando-se os valores obtidos para o controle (T0), observa-se que os resultados para o solo LV nas três coletas $(94,41$,
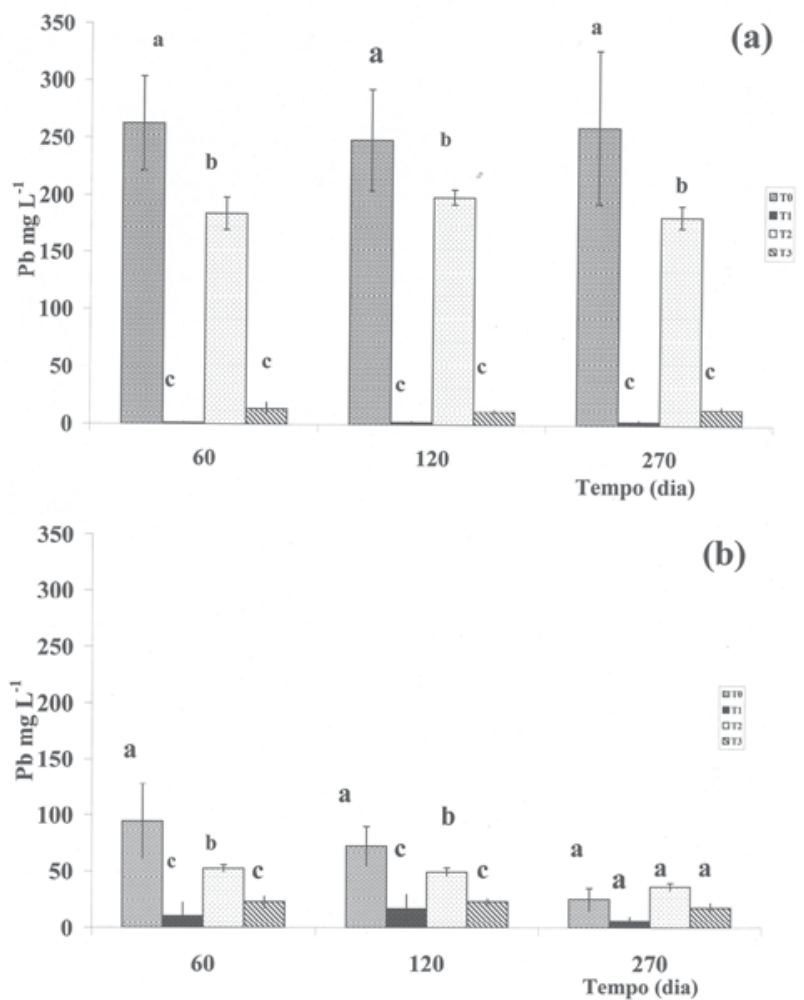

Figura 4. Concentrações de Pb após extração por TCLP em amostras de solos LA (a) e LV (b) coletadas em 60, 120 e 270 dias após os tratamentos. Letras minúsculas diferentes representam diferença significativa $(P<0,05)$ pelo teste de Tukey

72,4 e $25,0, \mathrm{mg} \mathrm{kg}^{-1}$ ) são muito inferiores àqueles obtidos para o LA $\left(262,5,248,4\right.$ e $\left.260,3 \mathrm{mg} \mathrm{kg}^{-1}\right)$. Adicionalmente pode-se notar que a concentração de chumbo extraída do LV decresce à medida que se aumenta o tempo da contaminação. Como os solos controle não receberam tratamento com fosfato, pode-se depreender que esse decréscimo deve-se à atenuação natural relativa a alguma fração da composição do solo, possivelmente, óxidos de ferro e manganês e matéria orgânica. Além disso, o tempo de contato mais prolongado (270 dias) parece favorecer os mecanismos naturais devido às próprias características do solo LV, o que o torna tão eficiente no decréscimo da concentração de chumbo quanto à adição de fosfatos.

\section{Teste ecotoxicológico com Daphnias pulex}

A partir dos resultados dos testes de ecotoxicidade com Daphnia foi estabelecido o fator de toxicidade (FT) para cada lixiviado. Todas as amostras apresentaram-se altamente tóxicas para Daphnia, entretanto, nas amostras de solos tratadas com fosfatos foi verificado decréscimo da toxicidade (dados não apresentados). Além disso, houve uma diminuição da toxicidade do extrato com o tempo de tratamento. Também pode ser observado que o fator de toxicidade para o solo LV foi sensivelmente menor que o do LA e diminuiu significativamente com o tempo, reforçando a idéia da existência, neste tipo de solo, de um mecanismo de imobilização paralelo ao do fosfato.

Nos estudos de materiais tóxicos, geralmente, associa-se a dose a uma resposta. Neste trabalho a resposta foi relacionada à imobilidade dos organismos (Daphnias) após 24 h de exposição na solução teste em diferentes concentrações (dose). A Figura 5 (a e b) 
estabelece a relação da exposição dos indivíduos na solução-teste e o efeito causado (imobilidade) para diferentes concentrações.
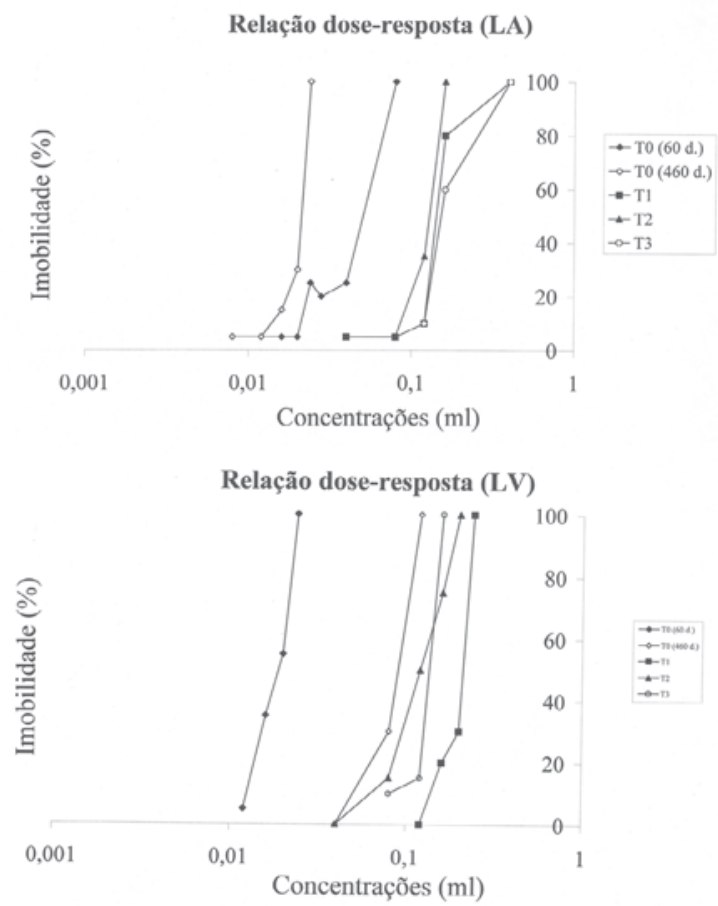

Figura 5. Relação dose-resposta nos solos LA (a) e LV (b) para diferentes doses (concentrações da solução-teste). T0-60 dias $(\diamond)$; T0-460 dias $(\diamond)$; $T 1$ ( $\mathbf{\square}) ; T 2$ ( $\mathbf{\Delta})$ e T3 (O)

O resultado da relação dose-resposta entre diferentes concentrações da solução-teste mostrou comportamento diferenciado para os dois solos. No solo LA o T0 (460 dias) foi o que apresentou maior toxicidade, pois, a partir de $0,008 \%$ de concentração da soluçãoteste verificou-se imobilidade dos organismos. No T3 o efeito só começa a ser percebido a partir de $0,12 \%$ de concentração, podendo ser considerado o tratamento que expressou menor toxicidade para o solo LA. O T1 e o T2 manifestaram comportamentos semelhantes.

No solo LV a maior toxicidade foi observada para T0 (60 dias), a partir de $0,012 \%$ de concentração da solução-teste. O T1 foi o tratamento que expressou menor toxicidade, pois, só começou a apresentar resposta a partir de $0,12 \%$ de concentração. O T0 (460 dias) e o T2 manifestam efeitos a partir de 0,04 e o T3 com $0,08 \%$ de concentração.

\section{Extração seqüencial}

Os dados obtidos a partir da extração seqüencial também foram submetidos à análise de variância (ANOVA) utilizando o programa estatístico $\mathrm{SAEG}^{17}$ com base nos valores absolutos (mg de $\mathrm{Pb} \mathrm{kg}^{-1}$ de solo) e nos valores percentuais de participação de cada fase no total extraído. Quando a variância foi significativa no teste F, utilizou-se o teste de comparação de médias Scott Knott, o qual faz agrupamento estatístico a partir das médias. ${ }^{18}$

A análise de variância seguiu o modelo de delineamento experimental conduzido em um fatorial composto de dois tratamentos. ${ }^{22} \mathrm{O}$ tratamento A com dois solos de mineralogia distinta, onde o solo LA representa o predomínio de caulinita e o solo LV, a maior participação de matéria orgânica e óxidos de Fe e Mn. O tratamento $\mathrm{B}$ foi referente às amostras contaminadas e tratadas com as diferentes fontes de fosfato, além da amostra controle, ou seja, aquela que não sofreu nenhum processo de remediação do chumbo. As análises deste estudo consideraram três coletas de subamostras de solo nos seguintes tempos: 60, 120 e 270 dias após os tratamentos com fósforo.

As análises de variância mostraram diferenças significativas provocadas pelo tipo de solo, tratamentos utilizados e suas interações. Para efeito de discussão, as frações foram separadas em dois grupos: o primeiro F123 representando as frações que contêm as formas mais solúveis e o segundo F456, as frações contendo as formas menos solúveis, ou seja, aquelas que necessitam tratamento químico mais drástico para serem solubilizadas.

Os resultados obtidos são apresentados na Figura 6 (a, b e c). Como pode ser observado, todos os tratamentos mostraram-se eficientes em transferir o chumbo das frações mais disponíveis para as menos disponíveis. No período estudado, o tratamento com $\mathrm{H}_{3} \mathrm{PO}_{4}$
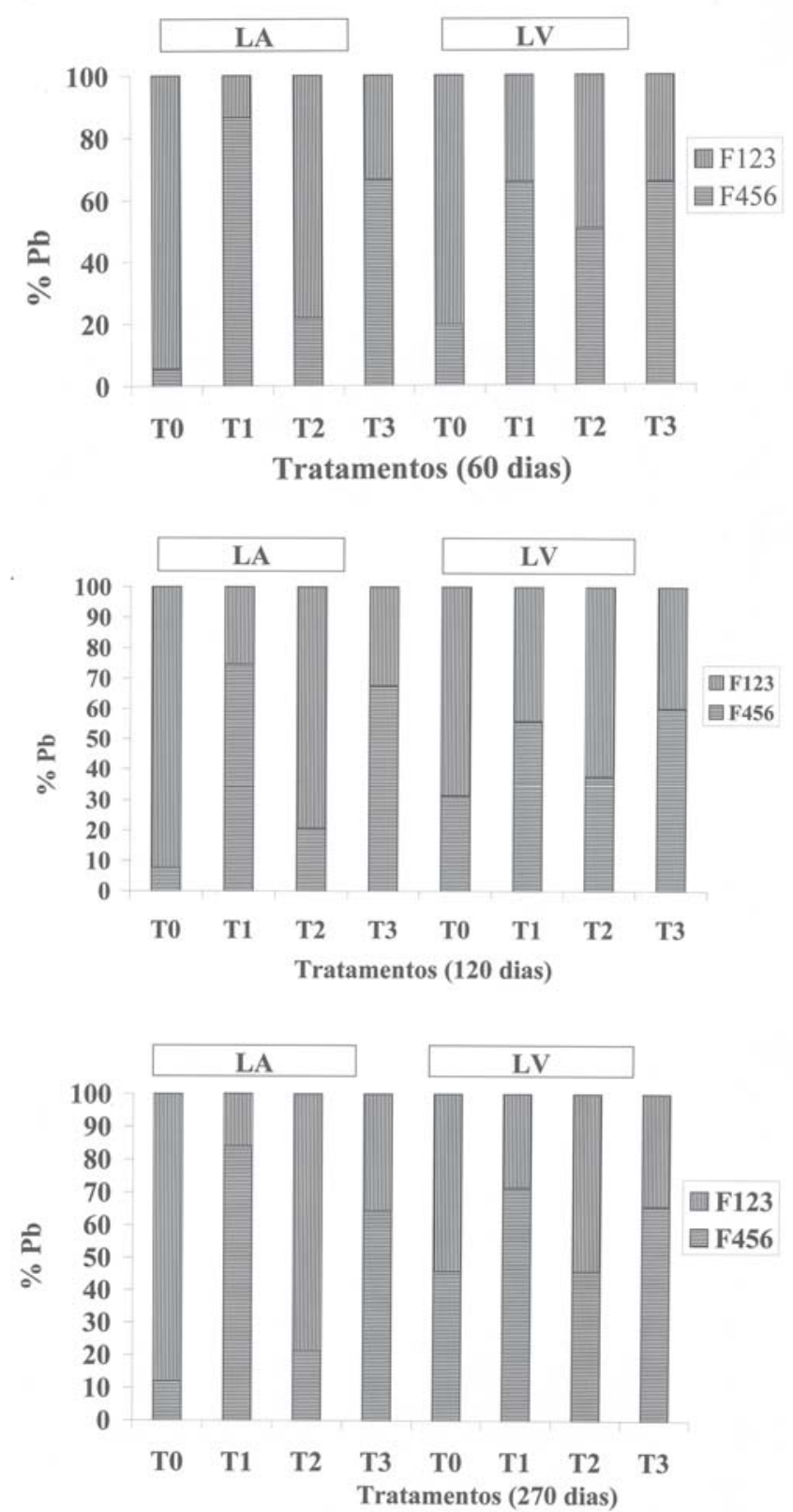

Figura 6. Extração seqüencial após 60 (a), 120 (b) e 270 (c) dias da realização dos tratamentos (TO - solo sem tratamento; $\mathrm{T1}-\mathrm{H}_{3} \mathrm{PO}_{4} ; \mathrm{T} 2$ - rocha; $\mathrm{T3}$ $\mathrm{H}_{3} \mathrm{PO}_{4}$ e rocha) 
mostrou-se o mais eficiente, seguindo-se o tratamento com a mistura de $\mathrm{H}_{3} \mathrm{PO}_{4}$ e rocha e, finalmente, com a rocha fosfatada. Isto era esperado uma vez que a cinética envolvida na imobilização pela rocha é muito mais lenta que a dos outros tipos de tratamento utilizados. Entretanto, a composição química do solo mostrou-se igualmente importante na transformação das formas solúveis para as menos solúveis. A transformação das formas solúveis em menos solúveis é acentuadamente maior no latossolo vermelho que no amarelo, mostrando a importância do tipo de solo contaminado no tratamento. Isso sugere que alguns tipos de solos apresentam mecanismos naturais capazes de auxiliarem a imobilização do chumbo, como discutido anteriormente nos resultados de TCLP. No caso dos solos estudados isto pode ser explicado pelos valores mais elevados de $\mathrm{Fe}_{2} \mathrm{O}_{3}, \mathrm{MnO}$ e matéria orgânica (Tabela 1) no LV que competem com o fósforo pelo chumbo. $\mathrm{O}$ mesmo não ocorre com LA que é essencialmente caulinítico e, portanto, pouco reativo.

\section{CONCLUSÕES}

A remediação de solos contaminados por chumbo utilizando fósforo provoca uma alteração na forma geoquímica do metal, reduzindo a sua solubilidade. Contudo, a capacidade de imobilização do chumbo resultante de sua reação com fosfatos é dependente de vários fatores, tais como tipo da fonte e da aplicação do fósforo, tipo de solo, concentração do metal e do tempo após aplicação dos fosfatos.

Os tratamentos realizados mostraram-se eficientes na diminuição da solubilidade do $\mathrm{Pb}$. Comparativamente, os tratamentos com $\mathrm{H}_{3} \mathrm{PO}_{4}$ (T1 e T3) foram os mais eficientes em converter o chumbo para as formas menos disponíveis. Os resultados obtidos mostram claramente que a composição do solo é um fator de grande importância na transformação de formas químicas solúveis de chumbo em forma menos solúveis que são menos biodisponíveis. A identificação das substâncias responsáveis por esta imobilização abre novas perspectivas para o tratamento de solos contaminados com chumbo.

Os testes ecotoxicológicos mostraram que todos os tratamentos foram eficientes em diminuir o nível de toxicidade inicial. Entretanto, mesmo após os tratamentos realizados os solos ainda apresentavam elevada toxicidade, demonstrando que as metodologias utilizadas precisam ser otimizadas para se tornarem toxicologicamente mais efetivas. Neste aspecto, a utilização de frações naturalmente presentes em alguns tipos de solos, por exemplo no LV, para remediação de áreas contaminadas pelo chumbo torna-se altamente atraente.

\section{AGRADECIMENTOS}

Ao $\mathrm{CNPq}$ pelo apoio recebido e também aos pesquisadores $\mathrm{D}$. Baptista e D. Paiva pela realização dos testes de toxicidade.

\section{REFERÊNCIAS}

1. Davies, B. E. Em Heavy Metals in Soils Lead; Alloway, B. J., ed.; Blackie and Son Ltd: New York, 1990, cap. 9.

2. Manecki, M.; Bogucka, A.; Badja, T.; Borkiewicz, O.; Environ. Chem. Lett. 2006, 3, 178.

3. Paoliello, M. M. B.; Capitani, E. M.; Cunha, F. G.; Matsuo, T.; Carvalho, M. F.; Sakuma A.; Figueiredo, B. R.; Environ. Res. A 2002, 88, 120.

4. Anjos, J. A. S. A.; Sánchez, L. E.; Bahia Análise \& Dados 2001, 10, 306.

5. http://www.cetesb.sp.gov.br/Noticias/002/04/10_ajax.asp, acessada em Abril 2007.

6. Papanikolaou, N. C.; Hatzidaki, E. G.; Belivanis, S.; Tzanakakis, G. N.; Tsatsakis, A. M.; Méd. Sci. Monit. 2005, 11, 329.

7. Costa, L. G.; Aschner M.; Vitalone A.; Syversen T.; Soldin O. P.; Annu. Rev. Pharmacol. Toxicol. 2004, 44, 92.

8. Baird, N. C.; Química Ambiental, 2a ed., Editora Bookman: Porto Alegre, 2002.

9. Ma, L.; Rao, G. N.; J. Environ. Qual. 1997, 26, 788; Cao, X.; Ma, L. Q.; Chen, M.; Singh, S. P.; Harris, W. G.; Environ. Sci. Technol. 2002, 36, 5296; Cao, R. X.; Ma, L. Q.; Chen, M.; Singh, S. P.; Harris, W. G.; Environ. Pollut. 2003, 122, 19; Chen, X.; Wright, J. V.; Conca, J. L.; Peurrung, L. M.; Environ. Sci. Technol. 1997, 31, 624.

10. EMBRAPA; Manual de métodos de análise do solo, Rio de Janeiro, 1997.

11. Deer, W. W. A.; Howie, R. A.; Zussma, N. J.; Minerais constituintes das rochas: uma introdução, Fundação Calouste Gulbenkian: Lisboa, 1981.

12. Mavropoulos, E.; Rocha, N. C. C.; Moreira, J.; Bertolino, L. C.; Rossi, A. M.; J. Braz. Chem. Soc. 2005, 16, 62.

13. http://www.epa.gov/sw-846/pdfs/1311.pdf, acessada em Novembro 2003.

14. Knie, J. L. W.; Lopes, E. W. B.; Testes ecotoxicológicos. Métodos, técnicas e aplicações, FATMA/GTZ: Florianópolis, 2004.

15. Morrow, D. A.; Gintautas, P. A.; Weiss, A. D.; Piwoni, M. D.; Metals Speciation in Soils: A Review of Methodologies, Prepared for U.S. Army Corps of Engineers, UK, 1996; Lã, O. R.; Barra, C. M.; do Amaral Sobrinho, N. M. B.; Mazur, N.; Velloso, A. C. X.; Quim. Nova 2003, 26, 323.

16. Tessier, A.; Campbell, P. G. C.; Bisson, M.; Anal. Chem. 1979, 51, 844.

17. SAEG 6; Sistema para análise estatística e genética, V 9, Universidade Federal de Viçosa: Viçosa, 2005.

18. Fernandez, G. C. J.; HortSci. 1992, 27, 297.

19. Hyötyläinen, T.; Oikari, A.; Chemosphere 1999, 38, 1135.

20. Suzuki, Y.; Takeuchi Y.; J. Chem. Eng. Jpn. 1994, 27, 571.

21. Ma, Q. Y.; Traina, S. J.; Logan, T. J.; Ryan, J. A.; Environ. Sci. Technol. 1993, 27, 1803.

22. Gomes, F. P.; A estatística moderna na pesquisa agropecuária, $3^{\mathrm{a}} \mathrm{ed}$, POTAFOS: Piracicaba, 1987. 